\title{
Verification of Hybrid Systems Based on Counterexample-Guided Abstraction Refinement ${ }^{\star}$
}

\author{
Edmund Clarke ${ }^{1}$, Ansgar Fehnker ${ }^{2}$, Zhi Han ${ }^{2}$, Bruce $\mathrm{Krogh}^{2}$, Olaf Stursberg ${ }^{2,3}$, and \\ Michael Theobald ${ }^{1}$ \\ ${ }^{1}$ Computer Science, Carnegie Mellon University, Pittsburgh, PA \\ 2 Electrical and Computer Engineering, Carnegie Mellon University, Pittsburgh, PA \\ 3 Process Control Lab, University of Dortmund, Germany
}

\begin{abstract}
Hybrid dynamic systems include both continuous and discrete state variables. Properties of hybrid systems, which have an infinite state space, can often be verified using ordinary model checking together with a finite-state abstraction. Model checking can be inconclusive, however, in which case the abstraction must be refined. This paper presents a new procedure to perform this refinement operation for abstractions of infinite-state systems, in particular of hybrid systems. Following an approach originally developed for finite-state systems [1]2], the refinement procedure constructs a new abstraction that eliminates a counterexample generated by the model checker. For hybrid systems, analysis of the counterexample requires the computation of sets of reachable states in the continuous state space. We show how such reachability computations with varying degrees of complexity can be used to refine hybrid system abstractions efficiently. A detailed example illustrates our counterexample-guided refinement procedure. Experimental results for a prototype implementation of the procedure indicate its advantages over existing methods.
\end{abstract}

\section{Introduction}

Hybrid systems are formal models that include both continuous and discrete state variables. With the increasing use of hybrid systems to design embedded controllers for complex systems such as manufacturing processes, automobiles, and transportation networks, there is an urgent need for more powerful analysis tools, especially for safety critical applications. Tools developed so far for automated analysis of hybrid systems are restricted to low-dimensional continuous dynamics [3]. The reason for this limitation is the difficulty of representing and computing sets of reachable states for continuous dynamic systems. Recent publications have proposed two general approaches to deal

${ }^{\star}$ This research was supported by the Defense Advanced Research Project Agency (DARPA) MoBIES project under contracts no. F3361500C1701 and F33615-02-C-0429, by the Army Research Office (ARO) under contract no. DAAD19-01-1-0485, by the National Science Foundation (NSF) under grants no. CCR-0121547 and CCR-0098072. The views and conclusions contained in this document are those of the authors and should not be interpreted as representing the official policies, either expressed or implied, of DARPA, ARO, NSF, the U.S. Government or any other entity. 
with the complexity of hybrid system analysis, namely, modular analysis (e.g., [45]) and abstraction (e.g., [6,78]). This paper focuses on the latter approach.

Abstraction maps a given model into a less complex model that retains the behaviors of interest [6]. In the context of hybrid system verification, abstraction transforms the inherently infinite state system into a finite-state model [78]. Existing tools often do not consider the property itself when building an abstract model. Rather, an abstract representation is constructed for the entire hybrid system using a degree of detail which seems to be appropriate. If the abstraction is not appropriate to analyze the property, the whole abstraction process is started again, or the abstract model is globally refined [9].

As an alternative, we suggest a procedure that (a) starts from a coarse abstract model and a safety property, (b) identifies parts of the hybrid system which potentially violate the property, and (c) iteratively refines the abstract model until verification reveals whether or not the property in question is satisfied. A framework that follows this general scheme of abstraction, refinement, and analysis, is counterexample-guided abstraction refinement $(C E G A R)$ [1 10[2]: For a given system the initial abstraction leads to a conservative model that is guaranteed to include all behaviors of the original system. Model checking is then applied to the abstract model. If the property is violated, the model checker produces a counterexample as an execution path for the abstract model for which the property is not true. If the counterexample corresponds to a behavior of the original system, then the property does not hold for the original system. Otherwise, the information provided by the counterexample is then used to refine the abstract model, i.e., some detail is added to the abstract model in order to obtain a more accurate, yet conservative, representation of the original model. In particular, the refined model is constructed so that it is guaranteed to exclude the spurious counterexample. The procedure of alternating between model checking and refinement is continued until the property is confirmed or refuted.

This procedure has recently been applied successfully to finite discrete systems in a variety of domains, particularly for the verification of digital circuits [110]. Earlier work that is based on the use of counterexamples includes the localization reduction in the context of concurrent systems [2], and recent work has applied the technique to the verification of C-programs [1112]. Another related abstraction refinement approach for programs [13] is not based on counterexamples but uses backward and forward reachability to decide how to refine an abstract model.

This paper makes two important contributions. First, we extend counterexampleguided model refinement to infinite-state systems. Second, we show how our new approach can be applied to hybrid systems, which include both continuous and discrete state variables and thus have an infinite-state space. We provide effective means of coping with the difficulties of computing reachable sets for infinite state systems. In particular, we employ reachable set computations with varying degrees of complexity to refine hybrid system abstractions efficiently. This flexibility cannot easily be achieved with other verification tools for hybrid systems. We note that using counterexamples to guide generation of discrete abstractions is being pursued independently by Alur et al. at University of Pennsylvania [14].

The paper is structured as follows. Section 2 presents preliminaries on abstraction and counterexample-guided refinement. In Section 3 we describe a new verification approach that refines abstract models of infinite state systems based on counterexamples. 
We introduce hybrid systems in Section 4, and apply our new verification approach to hybrid systems in Section 5. Section 6 presents conclusions.

\section{Preliminaries}

We introduce the notions of abstraction and counterexample-guided refinement in a general setting for infinite state systems. The type of model we are working with throughout the section is a transition system defined as follows:

Definition 1 Transition System. A transition system is a 3-tuple $T S=\left(S, S_{0}, E\right)$ with a (possibly infinite) state set $S$, an initial set $S_{0} \subset S$, and a set of transitions $E \subset S \times S$.

Given two transition systems $A$ and $C, A$ is said to be an abstract model of $C$ if the following relation can be established.

Definition 2 Abstraction. A transition system $A=\left(\hat{S}, \hat{S}_{0}, \hat{E}\right)$ with a finite set of states $\hat{S}$ is an abstract model of a transition system $C=\left(S, S_{0}, E\right)$, denoted $A \succeq C$, if there exists an abstraction function $\alpha: S \rightarrow \hat{S}$ such that:

- the initial set is $\hat{S}_{0}=\left\{\hat{s}_{0} \mid \exists s_{0} \in S_{0}: \hat{s}_{0}=\alpha\left(s_{0}\right)\right\}$

- and $\hat{E} \supseteq\left\{\left(\hat{s}_{1}, \hat{s}_{2}\right) \mid \exists s_{1}, s_{2} \in S:\left(s_{1}, s_{2}\right) \in E, \hat{s}_{1}=\alpha\left(s_{1}\right), \hat{s}_{2}=\alpha\left(s_{2}\right)\right\}$.

Sometimes the term simulation is used in the literature to describe the abstraction relation. In contrast to the definitions of abstraction in [110], Defn. 2] allows that $A$ includes spurious transitions, i.e., the set $\hat{E}$ may contain elements that do not correspond to transitions in $C$. As a consequence the abstraction function in Defn. 2 does not uniquely define $A$. Spurious transitions arise in the construction of abstractions of hybrid systems because in most cases sets of reachable states for continuous systems can not be represented and computed exactly.

Abstract models will be used to analyze properties of a given transition system. Throughout the paper, we will call the given system $C$ the concrete system.

In order to construct a more detailed model from a given abstract model, we define the following concept of model refinement.

Definition 3 Refinement of Abstract Models. Given a concrete system $C=\left(S, S_{0}, E\right)$ and an abstract model $A=\left(\hat{S}, \hat{S}_{0}, \hat{E}\right)$ such that $C \preceq A$, with abstraction function $\alpha: S \rightarrow \hat{S}$, a model $A^{\prime}=\left(\hat{S}^{\prime}, \hat{S}_{0}^{\prime}, \hat{E}^{\prime}\right)$ is called a refined abstract model of $C$ with respect to $A$ if two abstraction functions $\alpha^{\prime}: S \rightarrow \hat{S}^{\prime}$ and $\alpha^{\prime \prime}: \hat{S}^{\prime} \rightarrow \hat{S}$ exist, i.e., $C \preceq A^{\prime} \preceq A$.

A property is verified for the concrete model $C$ using an abstract model $A$. In this paper we will consider the verification of safety properties, defined as follows.

Definition 4 Safety. Given a transition system $T S=\left(S, S_{0}, E\right)$, let the set $B \subset S$ specify a set of bad states such that $S_{0} \cap B=\emptyset$. We say that $T S$ is safe with respect to $B$, denoted by $T S \models \mathbf{A G} \neg B$ iff there is no path in the transition system from an initial state in $S_{0}$ to a bad state in $B$. Otherwise we say $T S$ is unsafe, denoted by $T S \forall \forall \mathbf{A G} \neg B$. 
Definition 5 Counterexamples. A path $\sigma=\left(s_{0}, s_{1}, \ldots, s_{m}\right)$ of $T S=\left(S, S_{0}, E\right)$ with $s_{m} \in B$ is called a counterexample of $T S$ with respect to the safety property $T S \models$ $\mathbf{A G} \neg B$. Given a concrete transition system $C$, an abstract transition system $A$, and a counterexample $\sigma$ in $C$, we say that $\hat{\sigma}=\left(\hat{s}_{0}, \hat{s}_{1}, \hat{s}_{2}, \ldots, \hat{s}_{m}\right)$ is the corresponding abstract counterexample of the abstract system $A$, if $\hat{s}_{i}=\alpha\left(s_{i}\right)$ holds for all $i \in$ $\{0, \ldots, m\}$. Given a counterexample $\hat{\sigma}$ of $A, \sigma$ is called a corresponding concrete counterexample if $\hat{s}_{i}=\alpha\left(s_{i}\right)$ and $\left(s_{i}, s_{i+1}\right) \in E$. If a counterexample $\hat{\sigma}$ of $A$ has no corresponding concrete counterexample for $C, \hat{\sigma}$ is called a spurious counterexample. $\diamond$

Lemma 1. Given a concrete model $C=\left(S, S_{0}, E\right)$, and an abstract model $A=$ $\left(\hat{S}, \hat{S}_{0}, \hat{E}\right)$ of $C$ with an abstraction function $\alpha$, let $B \subseteq S$, and $\hat{B}=\{\hat{b} \mid \exists b \in$ $B: \hat{b}=\alpha(b)\}$. If $A=\boldsymbol{A} \boldsymbol{G} \neg \hat{B}$, then $C \models \boldsymbol{A} \boldsymbol{G} \neg B$.

If $A \models \mathbf{A G} \neg \hat{B}$ can be verified, it can immediately be concluded from Lemma 1 (i.e., without applying verification to the concrete system $C$ ) that $C \models \mathbf{A G} \neg B$. On the other hand, the converse of Lemma 1 with respect to the AG-property does not hold. If the verification of $A$ reveals $A \not \forall \mathbf{A G} \neg \hat{B}$, then we cannot conclude that $C$ is not safe with respect to $B$, since the counterexample for $A$ may be spurious. We call a method that checks whether or not a counterexample is spurious a validation method. If the validation method discovers that the counterexample is spurious, then the counterexample is used to refine $A$. We now introduce a scheme for counterexampleguided refinement of abstractions to verify safety properties for a given concrete model. The basic principle is to repeat the following sequence of steps until the property is verified or refuted [1]. The starting point is a concrete model $C$ and an abstract model $A$ (we propose in Sec. 5.1] one specific way to obtain an initial abstract model for hybrid systems). For a set $B \subseteq S$ of bad states for $\mathrm{C}$, we assume for simplicity that $\alpha(s) \in \hat{B}$ implies $s \in B$. The first step is then to analyze $A \models \mathbf{A G} \neg \hat{B}$ by model checking. If this property holds it can immediately be concluded from Lemma 1 that $C$ is safe, too. Otherwise a counterexample is obtained, and it must be validated whether it has a corresponding concrete counterexample in $C$. If there is a corresponding concrete counterexample, then the safety property does not hold for $C$. In the other case, i.e. the counterexample is spurious, the counterexample is used to refine the model $A$. That is, a new and more detailed model $A^{\prime}$ with $C \preceq A^{\prime} \preceq A$ is determined, which excludes the spurious counterexample.

The procedure of model checking, validation of the counterexample, and refinement of the abstract model is repeated until the safety property is proved or refuted for $C$. The pseudo-code in Fig. 1 1 summarizes this procedure:

The crucial steps in the CEGAR procedure are validation, refinement, and model checking. With respect to model checking, standard algorithms for AG-properties can be used [15].

The important step in validating a counterexample is the computation of successors of states. We define an operator succ that determines the successor states from a given set $\tilde{S} \subseteq S$ by $\operatorname{succ}(\tilde{S})=\{s \in S \mid \exists \tilde{s} \in \tilde{S}:(\tilde{s}, s) \in E\}$. This set may not be exactly computable for a given concrete model $C$, i.e. only over-approximations $\overline{\operatorname{succ}}(\tilde{S}) \supset$ $\operatorname{succ}(\tilde{S})$ may be available. We first assume that $\operatorname{succ}(\tilde{S})$ is computable. 
ALGORITHM: Counterexample-Guided Abstraction Refinement: CEGAR

INPUT: Concrete model $C$ and a set of bad states $B$

OUTPUT: $B$ is (or is not) reachable

Generate initial abstract model $A$ (bad states are called $\hat{B})$

Generate counterexample $\hat{\sigma}$ by model checking $A$ wrt. $\hat{B}$

WHILE $\hat{\sigma}$ exists DO

Validation of $\hat{\sigma}$

IF $\hat{\sigma}$ validated THEN terminate with "B reachable"

ELSE

Generate refined model $A^{\prime}$ using counterexample $\hat{\sigma}$

$A:=A^{\prime}$

Generate next $\hat{\sigma}$ by model checking $A$ wrt. $\hat{B}$ ENDIF

ENDDO

Terminate with "B not reachable"

Fig. 1. CEgAR: Scheme for verifying/falsifying $C \models \mathbf{A G} \neg B$ based on counterexample-guided abstraction refinement

A counterexample $\hat{\sigma}=\left(\hat{s}_{0}, \ldots, \hat{s}_{m}\right)$ of $A$ is then validated as follows: Let $S_{k}=$ $\alpha^{-1}\left(\hat{s}_{k}\right), k \in\{0, \ldots, m\}$ denote the set of concrete states corresponding to an element of $\hat{\sigma}$. The reachable parts of these sets are recursively defined by $S_{0}^{\text {reach }}:=S_{0}, S_{k}^{\text {reach }}:=$ $\operatorname{succ}\left(S_{k-1}^{r e a c h}\right) \cap S_{k}, k \in\{1, \ldots, m\}$. The counterexample is spurious iff $S_{k}^{r e a c h}=\emptyset$ applies for at least one $k$, and we say the counterexample is refuted. Otherwise, the counterexample is validated, and $B$ is reachable.

If the counterexample is refuted with $S_{k}^{\text {reach }}=\emptyset$, the model $A$ is refined to a new finite abstract model $A^{\prime}=\left(\hat{S}^{\prime}, \hat{S}_{0}^{\prime}, \hat{E}^{\prime}\right)$ (cf. Defn. 3]. The refined model should take into account that there are no concrete transitions from states in $S_{k-1}^{\text {reach }}$ to states in $S_{k}$. We therefore require that the set $\hat{E}^{\prime}$ of $A^{\prime}$ does not contain transitions in the set $\left\{\left(\alpha^{\prime}\left(s_{1}\right), \alpha^{\prime}\left(s_{2}\right)\right) \mid \exists s_{1} \in S_{k-1}^{r e a c h}, s_{2} \in S_{k}\right\}$. Thus, succeeding refined models will exclude previously explored counterexamples. A method for the refinement of abstract models for infinite-state systems will be presented in the next section.

\section{Refinement of Abstract Models for Infinite State Systems}

This section presents a specific method for refining an abstract model $A$ for an infinite state system. The main idea is to directly use the information obtained from the validation procedure to refine some abstract states: Assume that the abstract model includes a transition between $\hat{s}_{1}$ and $\hat{s}_{2}$, while the validation of the counterexample has revealed that only a subset of concrete states in $S_{2}:=\alpha^{-1}\left(\hat{s}_{2}\right)$ is reachable from concrete states in $S_{1}:=\alpha^{-1}\left(\hat{s}_{1}\right)$. In this case we refine $A$ by splitting $\hat{s}_{2}$ into two new states. The first one, denoted by $\hat{s}_{2}^{\text {reach }}$, represents the reachable subset of $S_{2}$, given by $S_{2}^{\text {reach }}:=$ $\operatorname{succ}\left(S_{1}\right) \cap S_{2}$. The second one, denoted by $\hat{s}_{2}^{c o m p}$, represents the complement of the reachable part, given by $S_{2}^{c o m p}:=S_{2} \backslash S_{2}^{\text {reach }}$. In addition, the abstraction function that maps concrete states to abstract ones has to be refined, too. 
Definition 6 Refinement by State Splitting. Given a concrete model $C=\left(S, S_{0}, E\right)$ and an abstract model $A=\left(\hat{S}, \hat{S}_{0}, \hat{E}\right)$ with an abstraction function $\alpha: S \rightarrow \hat{S}$. Let $\left(\hat{s}_{1}, \hat{s}_{2}\right) \in$ $\hat{E}$ be a transition of a counterexample $\hat{\sigma}$. Then, we define $\rho_{\text {split }}$ as a refinement function that maps $A, \alpha$, and $\left(\hat{s}_{1}, \hat{s}_{2}\right) \in \hat{E}$ onto the refined abstract model $A^{\prime}=\left(\hat{S}^{\prime}, \hat{S}_{0}^{\prime}, \hat{E}^{\prime}\right)$ and the refined abstraction function $\alpha^{\prime}: S \rightarrow \hat{S}^{\prime}$, i.e., $\left(A^{\prime}, \alpha^{\prime}\right)=\rho_{\text {split }}\left(A, \alpha,\left(\hat{s}_{1}, \hat{s}_{2}\right)\right)$, defined as follows:

$$
\begin{aligned}
& \text { - } \hat{S}^{\prime}=\left(\hat{S} \backslash \hat{s}_{2}\right) \cup\left\{\hat{s}_{2}^{\text {reached }}, \hat{s}_{2}^{\text {comp }}\right\} \\
& \text { - } \alpha^{\prime}(s)=\left\{\begin{array}{l}
\alpha(s) \text { if } s \notin S_{2} \\
\hat{s}_{2}^{\text {reach }} \text { if } s \in S_{2}^{\text {reach }} \\
\hat{s}_{2}^{\text {comp }} \text { if } s \in S_{2}^{\text {comp }}
\end{array}\right. \\
& \text { - } \hat{S}_{0}^{\prime}=\left\{\hat{s}^{\prime} \in \hat{S}^{\prime} \mid \alpha^{\prime \prime}\left(\hat{s}^{\prime}\right) \in \hat{S}_{0}\right\} \\
& \text { - } \hat{E}^{\prime}=\left\{\left(\hat{s}_{1}^{\prime}, \hat{s}_{2}^{\prime}\right) \in \hat{S}^{\prime} \times \hat{S}^{\prime} \mid \exists \hat{s}_{1}, \hat{s}_{2} \in \hat{S} \quad:\left(\hat{s}_{1}, \hat{s}_{2}\right) \in \hat{E} \wedge \hat{s}_{1}=\alpha^{\prime \prime}\left(\hat{s}_{1}^{\prime}\right) \wedge \hat{s}_{2}=\right. \\
& \left.\alpha^{\prime \prime}\left(\hat{s}_{2}^{\prime}\right)\right\} \backslash\left(\hat{s}_{1}, \hat{s}_{2}^{\text {comp }}\right)
\end{aligned}
$$

where $\alpha^{\prime \prime}: \hat{S}^{\prime} \rightarrow \hat{S}$ maps $\hat{s}^{\prime}$ onto itself if $\hat{s}^{\prime} \notin\left\{\hat{s}_{2}^{\text {reached }}, \hat{s}_{2}^{\text {comp }}\right\}$, and on $\hat{s}_{2}$ otherwise.

Lemma 2. Let $A=\left(\hat{S}, \hat{S}_{0}, \hat{E}\right)$ be an abstract model of $C=\left(S, S_{0}, E\right)$ with the abstraction function $\alpha: S \rightarrow \hat{S}$. For a given transition $\left(\hat{s}_{1}, \hat{s}_{2}\right) \in \hat{E}$, assume that $S_{2}^{\text {reach }} \neq \emptyset$ holds. Then, $\left(A^{\prime}, \alpha^{\prime}\right):=\rho_{\text {split }}\left(A, \alpha,\left(\hat{s}_{1}, \hat{s}_{2}\right)\right)$ satisfies $A \succeq A^{\prime} \succeq C$.

The idea of splitting an abstract state has also been considered by Jeannet et al. [13]. However, their method does not address hybrid systems, and it uses forward and backward reachability on the abstract model rather than counterexamples to decide which state to split. One advantage (among others) of a counterexample-based approach is that it terminates quickly when a discovered counterexample is not spurious and thus proving that the safety property does not hold for the concrete system.

As a next step, we consider the case where the set of successors of $S_{1}$ and the set $S_{2}$ are disjoint. In this case, we can simply omit the corresponding abstract transition.

Definition 7 Refinement by Eliminating a Transition. The function $\rho_{\text {purge }}$ is a refinement that maps an abstract model $A=\left(\hat{S}, \hat{S}_{0}, \hat{E}\right)$, an abstraction function $\alpha: S \rightarrow \hat{S}$ and a transition $\left(\hat{s}_{1}, \hat{s}_{2}\right) \in \hat{E}$ onto $A^{\prime}=\left(\hat{S}, \hat{S}_{0}, \hat{E}^{\prime}\right)$ with $\hat{E}^{\prime}=\hat{E} \backslash\left(\hat{s}_{1}, \hat{s}_{2}\right)$.

Lemma 3. Let $A=\left(\hat{S}, \hat{S}_{0}, \hat{E}\right)$ be an abstract model of $C=\left(S, S_{0}, E\right)$ with the abstraction function $\alpha: S \rightarrow \hat{S}$. For a given transition $\left(\hat{s}_{1}, \hat{s}_{2}\right) \in \hat{E}$, assume that $S_{2}^{\text {reach }}=\emptyset$ holds. Then, $A^{\prime}:=\rho_{\text {purge }}\left(A, \alpha,\left(\hat{s}_{1}, \hat{s}_{2}\right)\right)$ satisfies $A \succeq A^{\prime} \succeq C$.

Based on these results, we now present a more specific formulation of the CEGAR algorithm in Fig. 2. called INFINITE-STATE-CEGAR, which uses the functions $\rho_{\text {split }}$ and $\rho_{\text {purge }}$ for refinement.

Correctness of the algorithm is implied by the following two lemmas 1 . Note that termination of the algorithm cannot be guaranteed as the number of states in the concrete model may be infinite, and a finite abstract model to verify (or disprove) the given property may not exist.

\footnotetext{
${ }^{1}$ The proofs of all lemmas in the paper can be found in [16].
} 
ALGORITHM: Infinite-State-Cegar

INPUT: Concrete model $C$ and a set of bad states $B$

OUTPUT: $B$ is (or is not) reachable

Generate initial abstract model $A$ and abstraction function $\alpha$

$\hat{B}:=\alpha(B)$

Generate counterexample $\hat{\sigma}=\left(\hat{s}_{0}, \ldots, \hat{s}_{m}\right)$ by model checking of $A$ wrt. $\hat{B}$

$S_{0}^{\text {reach }}:=\alpha^{-1}\left(\hat{s}_{0}\right)$

WHILE $\hat{\sigma}$ exists DO

// validation of counterexample

$k:=0$

WHILE $S_{k}^{\text {reach }} \neq \emptyset$ AND $k<m$ DO

$$
k:=k+1
$$

ENDDO

$$
S_{k}^{\text {reach }}:=\operatorname{succ}\left(S_{k-1}^{\text {reach }}\right) \cap \alpha^{-1}\left(\hat{s}_{k}\right)
$$

// if counterexample is validated, then terminate, else refine

IF $S_{k}^{\text {reach }} \neq \emptyset$ THEN terminate with "B reachable"

ELSE

FOR $l=1, \ldots, k-1$

// split abstract state $\hat{s}_{l}$ into two: one that corresponds

// to $S_{l}^{\text {reach }}$ and one that corresponds to $\alpha^{-1}\left(\hat{s}_{l}\right) \backslash S_{l}^{\text {reach }}$

IF $S_{l}^{\text {reach }} \neq \alpha^{-1}\left(\hat{s}_{l}\right)$

$\operatorname{THEN}(A, \alpha):=\rho_{\text {split }}\left(A, \alpha, \hat{s}_{l-1}, \hat{s}_{l}\right)$

ENDIF

ENDFOR

// remove spurious transition between $\hat{s}_{k-1}$ and $\hat{s}_{k}$

$A:=\rho_{\text {purge }}\left(A, \alpha, \hat{s}_{k-1}, \hat{s}_{k}\right)$

Generate $\hat{\sigma}$ by model checking of $A$ wrt. $\hat{B}$ ENDIF

ENDDO

Terminate with "B not reachable"

Fig. 2. Infinite-State-Cegar.

Lemma 4. If the algorithm terminates with "B reachable", then $C \not \forall \boldsymbol{A G} \neg B$.

Lemma 5. If the algorithm terminates with "B not reachable", then $C \models A G \neg B$.

The proposed procedure of validating counterexamples and refining abstract models is based on the computation of successor states. Alternatively, one could formulate a similar algorithm that uses sets of predecessors, or even a combination of both as presented in [1] and [10].

The Infinite-STATE-CEgaR algorithm in Fig.2 is based on the assumption that sets of successor states are exactly computable. Lemma 5 holds, however, also if successor states are not exactly computable, and instead only over-approximations of the set of successor states can be computed. If only under-approximations of successor sets can be computed, Lemma 5 will not hold, but Lemma 4 will. 


\section{Hybrid Systems}

Hybrid systems are a class of infinite state systems that include both continuous and discrete state variables. This section presents hybrid automata, which are used to model hybrid systems. We illustrate these definitions with an example that models a simple car controller, which is also used in later sections to illustrate our new verification approach.

\subsection{Definition of Hybrid Automata}

Definition 8 Syntax of the Hybrid Automaton $H A$. A hybrid automaton is a tuple $H A=$ $\left(Z, z_{0}, X, i n v, X_{0}, T, g, j, f\right)$ where

- $Z$ is a finite set of locations with an initial location $z_{0} \in Z$.

- $X \subseteq \mathbb{R}^{n}$ is the continuous state space.

- inv $: Z \rightarrow 2^{X}$ assigns to each location $z \in Z$ an invariant of the form $i n v(z) \subseteq X$.

- $X_{0} \subseteq X$ is the set of initial continuous states. The set of initial hybrid states of $H A$ is thus given by the set of states $\left\{z_{0}\right\} \times X_{0}$.

- $T \subseteq Z \times Z$ is the set of discrete transitions between locations.

- $g: T \rightarrow 2^{X}$ assigns a guard set $g\left(\left(z_{1}, z_{2}\right)\right) \subseteq X$ to $t=\left(z_{1}, z_{2}\right) \in T$.

- $j: T \times X \rightarrow 2^{X}$ assigns to each pair $\left(z_{1}, z_{2}\right) \in T$ and $x \in g\left(\left(z_{1}, z_{2}\right)\right)$ a jump set $j\left(\left(z_{1}, z_{2}\right), x\right) \subseteq X$.

- $f: Z \rightarrow\left(X \rightarrow \mathbb{R}^{n}\right)$ assigns to each location $z \in Z$ a continuous vector field $f(z)$. We use the notation $f_{z}$ for $f(z)$. The evolution of the continuous behavior in location $z$ is governed by the differential equation $\dot{\chi}(t)=f_{z}(\chi(t))$. We assume that the differential equation has a unique solution for each initial value $\chi(0) \in X_{0}$. $\diamond$

The semantics of $H A$ is defined as a trace transition system. A state $(z, x)$ corresponds to a continuous state $x$ within location $z$. Two states, $\left(z_{1}, x_{1}\right)$ and $\left(z_{2}, x_{2}\right)$, are connected by a transition if and only if state $\left(z_{2}, x_{2}\right)$ can be reached from state $\left(z_{1}, x_{1}\right)$ by a continuous evolution within location $z_{1}$ followed by a discrete transition to location $z_{2}$.

Definition 9 Semantics of the Hybrid Automaton $H A$. The semantics of a Hybrid automaton $H A$ is a transition system $T T S=\left(S, S_{0}, E\right)$ with:

- the set of all hybrid states $(z, x)$ of $H A$,

$$
S=\bigcup_{z \in Z} \bigcup_{x \in \operatorname{inv}(z)}(z, x)
$$

- the set of initial hybrid states $S_{0}=\left\{z_{0}\right\} \times X_{0}$,

- transitions $\left(s_{1}, s_{2}\right) \in E$ with $s_{1}=\left(z_{1}, x_{1}\right), s_{2}=\left(z_{2}, x_{2}\right)$, iff there exists $\left(z_{1}, z_{2}\right) \in$ $T$ and a trajectory $\chi:[0, \tau] \rightarrow X$ for some $\tau \in \mathbb{R}^{>0}$ such that:

- $x_{1}=\chi(0), \chi(\tau) \in g\left(\left(z_{1}, z_{2}\right)\right)$,

- $x_{2} \in j\left(\left(z_{1}, z_{2}\right), \chi(\tau)\right)$,

- $\dot{\chi}(t)=f_{z_{1}}(\chi(t))$ for $t \in[0, \tau]$,

- $\chi(t) \in i n v\left(z_{1}\right)$ for $t \in[0, \tau]$,

- $x_{2} \in \operatorname{inv}\left(z_{2}\right)$. 
A path $\sigma=\left\{s_{0}, s_{1}, s_{2}, \ldots\right\}$ of TTS is called a trace of $H A$, and we refer to TTS as the trace transition system of $H A$.

Definition 10 Safety of a Hybrid Automaton. For a hybrid automaton $H A$ with a semantics as in Defn. 9 let $z_{b} \in Z \backslash\left\{z_{0}\right\}$ denote an unsafe location. $H A$ is said to be safe with respect to $z_{b}$, denoted by $T T S \models \mathbf{A G} \neg z_{b}$ iff for all traces $\sigma$ applies: $\nexists s \in \sigma$ with $s=\left(z_{b}, x\right)$ for some $x \in X$. We write $T T S \not \forall \mathbf{A G} \neg z_{b}$ otherwise.

The extension of the analysis task to multiple initial locations and/or multiple unsafe locations is straightforward but is omitted here for simplicity.

\subsection{Example}

As a motivating example, we use a simple controller that steers a car along a straight road. The car is assumed to drive at a constant speed $r=2$, and its motion is modeled by the distance $x$ ( $x=0$ corresponds to the middle of the road) from the middle of the road and the heading angle $\gamma(\gamma=0$ corresponds to moving straight ahead). Fig. 3 shows a scenario in which the car drives initially on the road. The controller is able to detect whether the car is on the left or right border (i.e. $x \leq-1, x \geq 1$ ) - whenever the car enters the left border, the controller forces it to turn right until the car is back on the road again. Then a left turn is initiated, and continued until the car is again going straight ahead in the direction of the road, i.e. when the heading is aligned with the road $(\gamma=0)$. A similar strategy is employed when the car enters the right border.
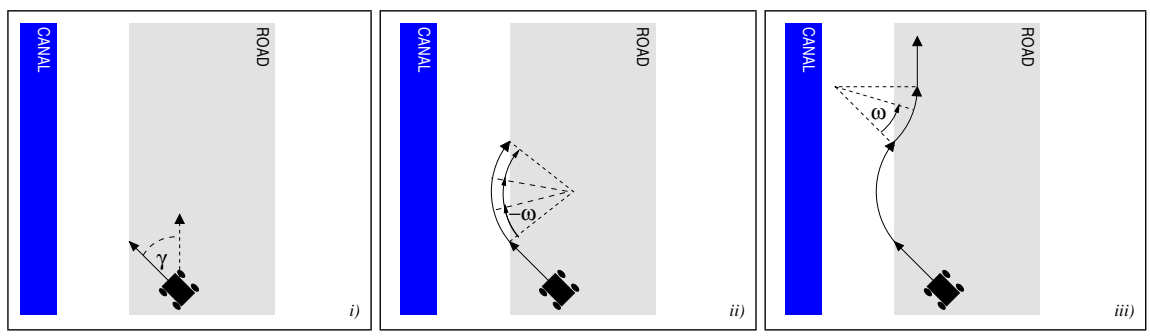

Fig. 3. $i$ ) Initially, the car drives on the road with heading angle $\gamma$. ii) If the controller detects that the car left the road, it corrects the heading by turning right to avoid the canal. iii) Once the car is back on the road, a left turn is initiated until the car moves straight again.

Fig.4/shows a hybrid automaton model of the controlled behavior for the car. Besides the position $x$ and the heading angle $\gamma$, the description includes an internal timer $c$, that the controller uses to time the steering manoeuvres. The differential equations for these three continuous variables depend on the location: we have $\dot{x}=-r \cdot \sin (\gamma)$ in all locations except of in_canal. The derivative of $\gamma$ varies when a border is reached. On the border the motion of the car describes an arc with the angular velocity $\dot{\gamma}=-\omega=-\pi / 4$ (or $\omega=\pi / 4$ respectively), i. e., the arc is part of a circle with radius $r / \omega$. The timer 
$c$ measures the time period which the car spends on a borders. In the correction modes the timer decreases with double rate, i.e., the correction takes half the time as the car was on the border before. Since the sign of $\dot{\gamma}$ is reversed when the car moves back on the road, the angle has the value zero when the correction mode is left $(c=0)$, i.e., the car moves then along the road. During this correction it might, however, happen that the other border is reached, which means that the controller then switches to the strategy of the corresponding location.

The three continuous variables are initialized to $-1 \leq x \leq 1$ (the car is on the road), $-\pi / 4 \leq \gamma \leq \pi / 4$, and $c=0$. It has to be verified for this set of initial states whether the given control strategy guarantees that the unsafe location in_canal $\left(z_{b}\right)$ is never reached. The following sections present how this task can be solved by abstraction-based and counterexample-guided verification.

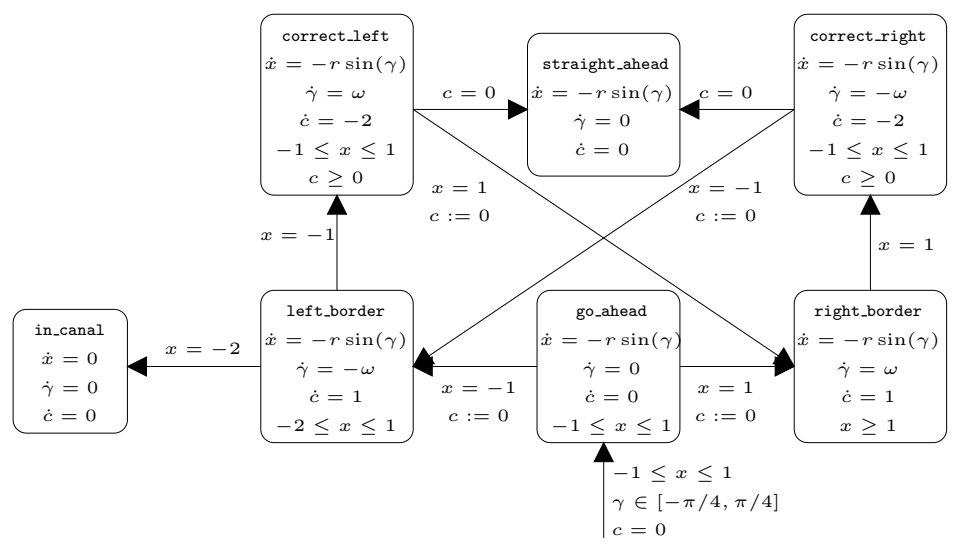

Fig. 4. Hybrid automaton that models the car steering example. Location in_canal has to be avoided. For each location, the continuous dynamics of the three variables $x, \gamma$ and $c$ is described by differential equations, and invariants are specified as inequalities. Guards and jumps are assigned to the transitions, e.g., a transition from location go_ahead to left_boarder is possible if the value of $x$ is 1 , and then the value of $c$ is set to zero.

\section{Refinement of Abstractions for Hybrid Systems}

This section applies the general concepts of Section 3 to the particular class of infinite state systems of hybrid systems.

We present specific solutions for the two crucial steps, the validation of counterexamples and the refinement of abstract models. The key to the validation step is the computation of successor states for a given set of states in the trace transition system. Starting from the initial set, the validation procedure computes the successors along the counterexample until either the unsafe location $z_{s p}$ is reached or a transition is determined to be spurious. The computation of sets of successors states is usually the most expensive step in hybrid system verification. Moreover, successor sets can be computed 
and represented exactly only for certain sub-classes of hybrid systems [17,18]. However, several approaches to over-approximate successor sets have been published, as e. g., approximations by orthogonal polyhedra [19], general polyhedra [20], projections to lower dimensional polyhedra [21], or ellipsoids [22]. Most of these approaches aim at providing an efficient way to obtain conservative but tight approximations.

The verification framework presented here can include different techniques to overapproximate the set of successors. The idea of using different methods is motivated by the trade-off between the accuracy and the computational complexity of different methods. If, e.g., a faster but maybe less accurate technique is sufficient to refute a counterexample, there is no need to use a computationally more expensive method.

In the following, we first describe how an initial abstraction for a hybrid automaton can be obtained, and then focus on the validation of counterexamples and the refinement based on the use of different methods for computing successor states.

\subsection{Abstraction of Hybrid Systems}

For the first step of INFINITE-STATE-CEGAR, the construction of an initial abstraction, we introduce one abstract state for each location of $H A$. This means that two hybrid states $\left(z_{i}, x_{i}\right)$ and $\left(z_{j}, x_{j}\right)$ of TTS are mapped to the same abstract state if and only if $z_{i}=z_{j}$. This rule applies for all but the initial location, which we split into two abstract states to separate the initial hybrid states in that location from the non-initial hybrid states: we introduce one abstract state $\hat{s}_{0}$ to represent all initial hybrid states of TTS, and another one $\left(\hat{s}_{0}^{\prime}\right)$ to represent the remaining hybrid states in location $z_{0}$.

Definition 11 Initial Abstraction of Hybrid Systems. Given a hybrid automaton $H A$ with $Z=\left\{z_{0}, z_{1}, \ldots, z_{n_{z}}\right\}$, let $S$ denote the set of hybrid states as defined in (1). For $i \in\left\{0,1, \ldots, n_{z}\right\}$, we define the abstraction function $\alpha: S \rightarrow \hat{S}$ by:

$$
\alpha\left(z_{i}, x\right)=\left\{\begin{array}{l}
\hat{s}_{0} \text { if } i=0 \wedge x \in X_{0} \\
\hat{s}_{0}^{\prime} \text { if } i=0 \wedge x \notin X_{0} \\
\hat{s}_{i} \text { otherwise }
\end{array}\right.
$$

and the initial abstract model $A=\left(\hat{S}, \hat{S}_{0}, \hat{E}\right)$ is defined by $(i \in\{0,1, \ldots, n\}, j \in$ $\left.\left\{0,1, \ldots, n_{z}\right\}\right)$ :

$-\hat{S}=\left\{\hat{s}_{0}^{\prime}, \hat{s}_{0}, \hat{s}_{1}, \ldots, \hat{s}_{n}\right\}$

- $\hat{S}_{0}=\left\{\hat{s}_{0}\right\}$

- $\hat{E}=\left\{\left(\hat{s}_{i}, \hat{s}_{j}\right) \mid\left(z_{i}, z_{j}\right) \in T\right\} \cup\left\{\left(\hat{s}_{0}^{\prime}, \hat{s}_{j}\right) \mid\left(z_{0}, z_{j}\right) \in T\right\} \cup\left\{\left(\hat{s}_{i}, \hat{s}_{0}^{\prime}\right) \mid\left(z_{i}, z_{0}\right) \in T\right\}$

The initial abstract model represents the discrete structure of the hybrid system without regarding the continuous dynamics and guards. It has to be shown that $A$ is indeed an abstract model of the underlying trace transition system, i.e., that it fulfills Defn.22:

Lemma 6. For $H A$ with trace transition system $T T S=\left(S, S_{0}, E\right)$, let $A=\left(\hat{S}, \hat{S}_{0}, \hat{E}\right)$ denote the initial abstract model for TTS. Then, $A \succeq T T S$. 
Example (cont.) Fig. 5depicts the initial abstract model of the hybrid system in Fig.4. It is a copy of the discrete part of the hybrid system, except that the initial location is divided into two parts: $\hat{s}_{0}$ represents the states in location go_ahead with $x \in[-1,1], \gamma \in[-\pi / 4, \pi / 4]$ and $c=0$, and $\hat{s}_{0}^{\prime}$ all other states in go_ahead. The abstract states $\hat{s}_{1}$ to $\hat{s}_{6}$ represent the hybrid states of the other locations (left_border, right_border, correct_left, correct_right, straight_ahead and in_canal, respectively).

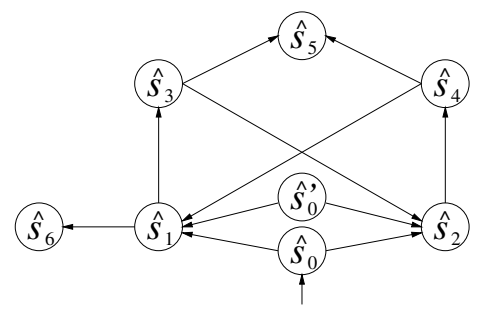

Fig 5. Initial abstract model of the hybrid system depicted in Fig. 4

\subsection{Over-Approximation of the Sets of Successors}

Computing sets of successor states is required in the validation and refinement steps. The goal is to use different over-approximations with different precisions and different computational needs. We first define an over-approximation operator of the successor relation for a tuple of sets of states. The operator conservatively approximates which states in the second set (target set) are successors of states in the first set (source set).

Definition 12 Over-approximation of successor states. Let $H A$ be a hybrid automaton with the trace transition system $T T S=\left(S, S_{0}, E\right)$, and let $A$ and $\alpha$ be defined as in Defn. 11 For a transition $\left(\hat{s}_{1}, \hat{s}_{2}\right) \in \hat{E}$ of $A$, we call $S_{1}:=\alpha^{-1}\left(\hat{s}_{1}\right)$ the set of hybrid source states and $S_{2}:=\alpha^{-1}\left(\hat{s}_{2}\right)$ the set of potential hybrid successor states. Then,

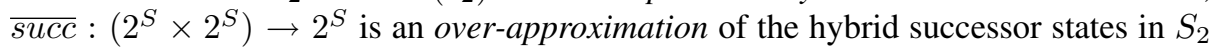
iff the following holds:

- $\overline{\operatorname{succ}}\left(S_{1}, S_{2}\right) \subseteq S_{2}$

- for all $s_{1} \in S_{1}$ and $s_{2} \in S_{2} \backslash \overline{\operatorname{succ}}\left(S_{1}, S_{2}\right),\left(s_{1}, s_{2}\right) \notin E$.

A possible explicit realization of the operator $\overline{s u c c}$ combines the following steps: (a) By approximating the continuous evolution for all states in $S_{1}$, the reachable subset of the guard set $g(t)$ is determined, where $t=\left(z_{1}, z_{2}\right) \in T$ is the transition of $H A$ that corresponds to the transition $\left(\hat{s}_{1}, \hat{s}_{2}\right) \in \hat{E}$ of $A$. Usually, this step is the most costly of the whole verification procedure; (b) the jump function $j(t, x)$ is applied to all hybrid states $\left(z_{1}, x\right)$ which are in the reachable subset of $g(t)$; (c) the image of $j(t, x)$ is intersected with the set $S_{2}$ of potential hybrid successor states.

Example (cont.) Our prototype implementation employs two different methods, $\overline{\text { succ }}_{\text {coarse }}$ and $\overline{\text { succ }}_{t i g h t}$, to over-approximate the set of successor states. For example, for the discrete transition from correct_right to left_border, we choose $S_{1}$ as subset of the plane $x=1$ for location correct_right, and $S_{2}$ as all states of location left_border that satisfy the invariant $-2 \leq x \leq-1$. The transition is not spurious, if there exists a trajectory that starts in $S_{1}$, and ends in $S_{2}$ without leaving the invariant of correct_right $(-1 \leq x \leq 1 \wedge c \geq 0)$.

The first method $\overline{s u c c}_{\text {coarse }}$ poses the existence question for a trajectory between $S_{1}$ and $S_{2}$ as an optimization problem. The distance between a trajectory and $S_{2}$ is defined as the minimum distance between all points on the trajectory and $S_{2}$. If the global minimum 
over all trajectories that start in $S_{1}$ is strictly greater than zero, then no successor state of $S_{1}$ exists in $S_{2}$. In this case $\overline{s u c c}_{\text {coarse }}$ returns an empty set. If the minimum distance is zero, at least one corresponding concrete path exists, and $\overline{s u c c}_{\text {coarse }}$ returns the complete set $S_{2}$ as an over-approximation of the set of successor states. In the considered example, the distance of the optimal trajectory to $S_{2}$ is greater than zero, and there is hence no trajectory from $S_{1}$ to $S_{2}{ }^{2}$. The second method $\overline{s u c c}_{t i g h t}$ computes polyhedra that enclose all trajectories that originate in $S_{1}$ [20]. The set of successor states $\overline{\operatorname{succ}}_{t i g h t}\left(S_{1}, S_{2}\right)$ is then obtained by intersecting the polyhedra with $S_{2}$. In the considered example, there are no successors of $S_{1}$ in $S_{2}$.

\subsection{Validation and Refinement}

INFINITE-STATE-CEGAR makes a clear distinction between the validation of a counterexample, and the refinement of the abstract model. For hybrid systems, we propose a slightly different approach, in which the steps of validation and refinement are interleaved. We assume to have a set of over-approximation techniques $\overline{s u c c}_{1}, \ldots, \overline{s u c c}_{p}$ that can (but do not need to) establish a hierarchy of coarse to tight approximations.

The algorithm for the combined validation and refinement steps of a counterexample is as follows. Let $\sigma=\left(\hat{s}_{0}, \ldots, \hat{s}_{m}\right)$ be a counterexample of the abstract model $A$. The algorithm consists of two nested loops. The outer loop corresponds to checking each transition of the counterexample. The inner loop applies each of the over-approximation techniques to the current transition of the counterexample, and, depending on the result, executes one of the two refinement operations: If an over-approximation technique $\overline{s u c c}$, $l \in\{1, \ldots, p\}$, reveals that the current transition is spurious, i.e. $S_{k}^{r e a c h}=\emptyset$, then the transition is removed from the abstract model by $\rho_{\text {purge }}$. When a transition is removed, the set of behaviors of $A$ does not include the current counterexample anymore, and thus the combined validation and refinement step is completed.

If on the other hand, $\overline{s u c c}_{l}$ returns a non-empty set $S_{\text {reach }}^{k}$ and this set is a true subset of the states corresponding to $\hat{s}_{k}$, the function $\rho_{\text {split }}$ divides $\hat{s}_{k}$ into two states $\hat{s}_{k}^{\text {reach }}$ and $\hat{s}_{k}^{c o m p}$ (cf. Defn.6). In this case however $\sigma=\left(\hat{s}_{0}, \ldots, \ldots, \hat{s}_{k-1}, \hat{s}_{k}^{r e a c h}, \hat{s}_{k+1} \ldots, \hat{s}_{m}\right)$ remains a counterexample of the refined model. Thus, the algorithm continues with the next transition $(k+1)$ until either $S_{k}^{r e a c h}=\emptyset$ or until the last transition of the counterexample is validated. There is some freedom in combining the steps of validation and refinement. One alternative is to apply the coarsest method for validation first to all transitions in the abstract counterexample, or to apply state splitting $\left(\rho_{\text {split }}\right)$ only based on the result of the most accurate approximation method $\overline{s u c c}_{p}$.

The proposed algorithm has two possible outcomes: either it is proved that a forbidden state cannot be reached or that there exists a counterexample that cannot be refuted. Since the validation procedure relies on over-approximations, it can not be guaranteed that this abstract counterexample corresponds to a concrete one. In this case, under-approximations of sets of successor states can possibly be used to prove that a counterexample exists: Assume that the procedure terminates with a counterexample $\sigma=\left(\hat{s}_{0}, \hat{s}_{1}, \ldots, \hat{s}_{k}, \ldots, s_{m}\right)$, no transition of which could be refuted. Similar to Defn. 12] we can define an under-approximation of successor states

\footnotetext{
${ }^{2}$ Illustrating figures can be found in [16]
} 


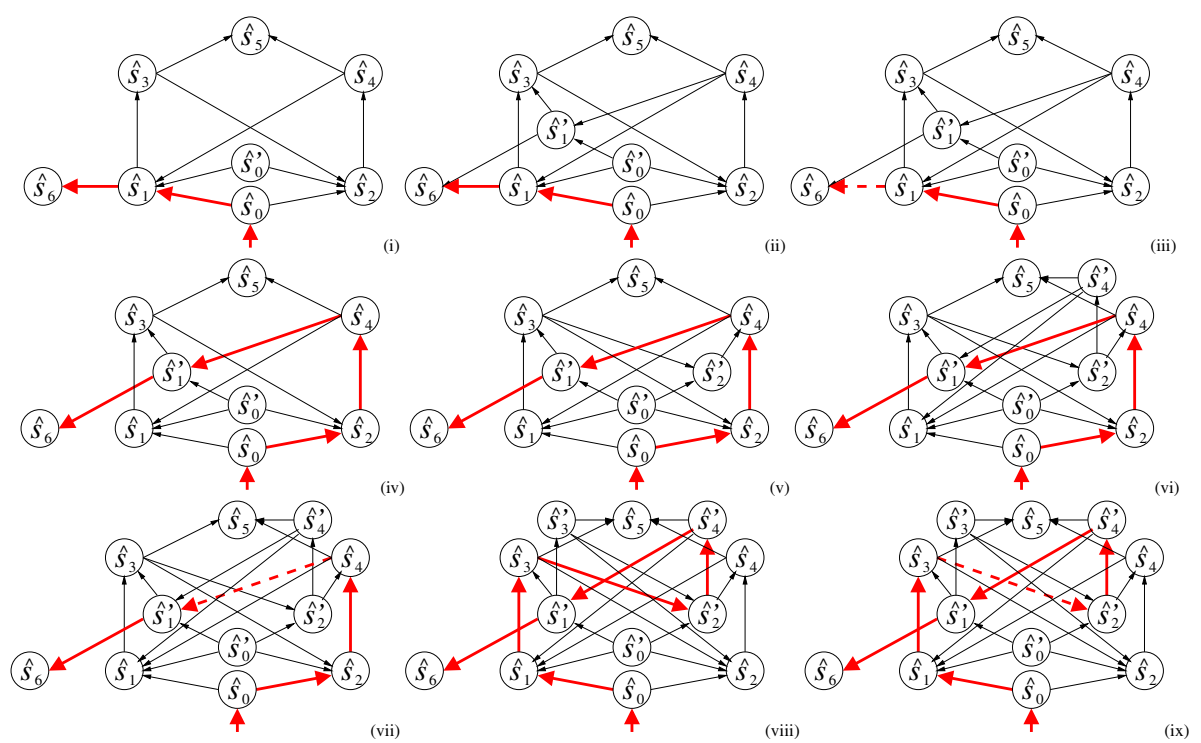

Fig. 6. Counterexample guided abstraction illustrated for the car steering problem.

$S_{k}^{\text {reach }}=\underline{\operatorname{succ}}\left(S_{k-1}^{\text {reach }}, \alpha^{-1}\left(\hat{s}_{k}\right)\right)$ which returns a set $S_{k}^{\text {reach }} \subseteq \alpha^{-1}\left(\hat{s}_{k}\right)$ for which it is ensured that it only contains true successors of $S_{k-1}^{r e a c h}$. If this operator is applied along the counterexample (from $k=1$ to $k=m$ ) and $S_{m}^{\text {reach }} \neq \emptyset$ applies, there exists at least one path for the hybrid system which violates the safety property.

Example (cont) The requirement that the hybrid model in Fig. 4 should never enter the location in_canal translates into the reachability question for state $\hat{s}_{6}$ of the abstract model in Fig. 5. The first counterexample for the initial abstract model is $\sigma_{1}=\left(\hat{s}_{0}, \hat{s}_{1}, \hat{s}_{6}\right)$ (see Fig. 6(i)). The validation procedure considers first the transition $\left(\hat{s}_{0}, \hat{s}_{1}\right)$ which corresponds to the transition between go_ahead and left_border in the hybrid automaton. As a first step, $\overline{\text { succ }}_{\text {coarse }}\left(S_{0}, \alpha^{-1}\left(\hat{s}_{1}\right)\right)$ is computed with the result that the minimum distance over all initial states is zero. This is obvious from the fact that those states of the initial set for which $x=-1$ enable the transition guard immediately. Thus, $\overline{\text { succ }}_{\text {coarse }}$ returns the entire invariant of location left_border as set $S_{2}$. The next step is to compute $S_{2}^{\text {reach }}=\overline{\operatorname{succ}}_{\text {tight }}\left(S_{0}, \alpha^{-1}\left(\hat{s}_{1}\right)\right)$. The algorithm then splits $\hat{s}_{1}$ such that $\hat{s}_{1}$ represents the set $S_{2}^{\text {reach }}$, and the new abstract state $\hat{s}_{1}^{\prime}$ represents $S_{2} \backslash S_{2}^{\text {reach }}$ (Fig.6(ii)). Since the counterexample has not been eliminated yet, the transition $\left(\hat{s}_{1}, \hat{s}_{6}\right)$ is considered next. $\overline{\text { succ }}_{\text {coarse }}$ finds that the minimal distance between the trajectories that start in $S_{2}^{\text {reach }}$, and the guard $x=-2$ is greater than zero. Thus, no trajectory reaches the guard, and the corresponding transition is removed (Fig. 6(iii)).

The procedure continues with the next counterexample $\sigma_{2}=\left(\hat{s}_{0}, \hat{s}_{2}, \hat{s}_{4}, \hat{s}_{1}^{\prime}, \hat{s}_{6}\right)$, as depicted in Fig. 6(iv). As for the first counterexample, the abstract state $\hat{s}_{2}$ is split into the states that are reachable from the initial set $S_{0}$, and the remainder (Fig. 6(v)). Then, the procedure moves one transition ahead and splits state $\hat{s}_{4}$ as a result of applying $\overline{\text { succ }}_{\text {tight }}$. The reachable part is represented by $\hat{s}_{4}$ in Fig.6(vi). Method $\overline{s u c c}_{\text {coarse }}$ then 
finds that one cannot reach any state that is represented by $\hat{s}_{1}^{\prime}$ from this set, and the transition $\left(\hat{s}_{4}, \hat{s}_{1}^{\prime}\right)$ can be deleted from $A$ (Fig. 6(vii)).

The final counterexample is $\sigma_{3}=\left(\hat{s}_{0}, \hat{s}_{1}, \hat{s}_{3}, \hat{s}_{2}^{\prime}, \hat{s}_{4}^{\prime}, \hat{s}_{1}^{\prime}, \hat{s}_{6}\right)$. The state $\hat{s}_{1}$ was already split for the first counterexample. Similarly to the procedure for the counterexample $\sigma_{2}$, state $\hat{s}_{3}$ is split as depicted in Fig. 6 (viii). It can be shown that transition $\left(\hat{s}_{3}, \hat{s}_{2}^{\prime}\right)$ is spurious, which eliminates the last counterexample (Fig.6(ix)). Consequently, the state $\hat{s}_{6}$ is not reachable, and thus the same applies for the location in_canal.

\subsection{Experimental Results}

Experimental results for a prototype implementation of the procedure indicate its advantages over existing methods. We compare INFINITE-STATE-CEGAR with a method based on breadth-first application of the successor operator $\overline{s u c c}_{t i g h t}$. Breadth-first application is the most prevalent method used for model checking hybrid systems. This approach needs 175 second cputime on a Pentium $4,1.4 \mathrm{GHz}$, to compute that location in_canal is not reachable. INFINITE-STATE-CEGAR together with only one of the two over-approximation methods, $\overline{s u c c}_{\text {tight }}$, takes 120 seconds. As in the case of the breadth-first methods, $99 \%$ of the cputime is spent on computing $\overline{\text { succ }}_{\text {tight }}$. If INFINITESTATE-CEgAR employs both approximation methods, then the time is cut in about half. The verification takes 68 seconds, of which 64 seconds are used to compute $\overline{s u c c}_{t i g h t}$, and 3 seconds to solve the optimization problems of $\overline{s u c c}_{\text {coarse }}$.

\section{Conclusions}

This paper presents a new method for using counterexamples to refine abstractions of hybrid systems. The principal alternative to verifying safety properties of hybrid systems by counterexample-guided verification is to compute the reachable states using a breadth-first application of the successor operator succ. It is apparent that the INFINITESTATE-CEGAR procedure can be faster than breadth-first reachability when the safety property does not hold for the concrete system, since in this case it is possible that the model checker will quickly find a true counterexample. On the other hand, if the safety property holds, refuting one counterexample may implicitly refute others. However, the INFINITE-STATE-CEGAR procedure may continue until all possible counterexamples have been explored (and indeed, may not terminate), which is in some cases equivalent to the breadth-first reachability computation. Nevertheless, INFINITE-STATE-CEGAR allows to use multiple methods for computing approximations to the successor states. Further evaluation of the INFINITE-STATE-CEGAR procedure and a comparison to breadth-first reachability and other alternatives is currently underway.

\section{References}

1. Clarke, E., Grumberg, O., Jha, S., Lu, Y., Veith, H.: Counterexample-guided abstraction refinement. In: CAV. Volume 1855 of LNCS, Springer (2000) 154-169

2. Kurshan, R.: Computer-Aided Verification of Coordinating Processes: The AutomataTheoretic Approach. Princeton University Press (1994) 
3. Silva, B., Stursberg, O., Krogh, B., Engell, S.: An assessment of the current status of algorithmic approaches to the verification of hybrid systems. In: IEEE Conf. on Decision and Control. (2001) 2867-2874

4. Henzinger, T., Minea, M., Prabhu, V.: Assume-guarantee reasoning for hierarchical hybrid systems. In: HSCC. Volume 2034 of LNCS, Springer (2001) 275-290

5. Frehse, G., Stursberg, O., Engell, S., Huuck, R., Lukoschus, B.: Modular analysis of discrete controllers for distributed hybrid systems. In: IFAC World Congress. (2002)

6. Alur, R., Henzinger, T., Lafferriere, G., Pappas, G.: Discrete abstractions of hybrid systems. Proceedings of the IEEE 88 (2000) 971-984

7. Alur, R., Dang, T., Ivancic, F.: Reachability analysis of hybrid systems via predicate abstraction. In: HSCC. Volume 2289 of LNCS, Springer (2002) 35-48

8. Tiwari, A., Khanna, G.: Series of abstractions for hybrid automata. In: HSCC. Volume 2289 of LNCS, Springer (2002) 465-478

9. Chutinan, A., Krogh, B.: Verification of infinite-state dynamic systems using approximate quotient transition systems. IEEE Transactions on Automatic Control 46 (2001) 1401-1410

10. Clarke, E., Gupta, A., Kukula, J., Strichman, O.: Sat based abstraction-refinement using ilp and machine learning techniques. In: CAV. LNCS, Springer (2002)

11. Ball, T., Majumdar, R., Millstein, T.D., Rajamani, S.K.: Automatic predicate abstraction of c programs. In: PLDI. SIGPLAN 36(5) (2001)

12. Henzinger, T., Jhala, R., Majumdar, R., Sutre, G.: Lazy abstraction. In: Symp. on Principles of Programming Languages, ACM Press (2002) 58-70

13. Jeannet, B., Halbwachs, N., Raymond, P.: Dynamic Partitioning in Analyses of Numerical Properties. In: Static Analysis Symposium (1999), 39-50

14. Alur, R., Dang, T., Ivančić, F.: Counter-Example Guided Predicate Abstraction of Hybrid Systems. In: TACAS, Springer (2003)

15. Clarke, E., Grumberg, O., Peled, D.: Model Checking. MIT Press (1999)

16. Clarke, E., Fehnker, A., Han, Z., Krogh, B., Ouaknine, J., Stursberg, O., Theobald, M.: Abstraction and Counterexample-Guided Refinement in Model Checking of Hybrid Systems. Technical report CMU-CS-03-104. Download from http://www.cs.cmu.edu/ theobald.

17. Lafferriere, G., Pappas, G., Yovine, S.: A new class of decidable hybrid systems. In: HSCC. LNCS 1569, Springer (1999) 103-116

18. Henzinger, T., Kopke, P., Puri, A., Varaiya, P.: What's decidable about hybrid automata? In: Symposium on Theory of Computing, ACM Press (1995) 373-382

19. Dang, T., Maler, O.: Reachability analysis via face lifting. In: HSCC. LNCS 1386 (1998)

20. Chutinan, A., Krogh, B.: Verification of polyhedral-invariant hybrid automata using polygonal flow pipe approximations. In: HSCC. LNCS 1569, Springer Verlag (1999) 76-90

21. Greenstreet, M., Mitchell, I.: Reachability analysis using polygonal projections. In: HSCC. LNCS 1569, Springer (1999) 103-116

22. Kurzhanski, A., Varaiya, P.: Ellipsoidal techniques for reachability analysis. In: HSCC. LNCS 1790, Springer (2000) 203-213 